\title{
UPLC-HRMS-Based Plasma Metabolomic Profiling of Novel Biomarkers by Treatment with KDZI in Cerebral Ischemia Reperfusion Rats
}

\author{
Chunguo Wang ${ }^{1,+}\left(\mathbb{D}\right.$, Chenyue Liu ${ }^{2,3,+}$, Min Wang ${ }^{2}$, Quantao $\mathrm{Ma}^{2}$, Yaqi $\mathrm{Li}^{2}$, Ting Wang ${ }^{1, *}$ \\ and Baosheng Zhao ${ }^{1, *}$ \\ 1 Beijing Research Institute of Chinese Medicine, Beijing University of Chinese Medicine, \\ Beijing 100029, China; wangcg1119@126.com \\ 2 School of Chinese Material Medica, Beijing University of Chinese Medicine, Beijing 100029, China; \\ liuchenyue633@163.com (C.L.); weme9916@163.com (M.W.); maquantao@bucm.edu.cn (Q.M.); \\ liyaqi@bucm.edu.com (Y.L.) \\ 3 School of Traditional Chinese Medicine, Beijing University of Chinese Medicine, Beijing 100029, China \\ * Correspondence: wangting1973@sina.com (T.W.); zhaobs1973@163.com (B.Z.); \\ Tel./Fax: +86-010-64286203 (T.W.); +86-010-64286894 (B.Z.) \\ + These authors contributed equally to this work.
}

Received: 2 May 2018; Accepted: 24 May 2018; Published: 30 May 2018

\begin{abstract}
Kudiezi injection (KDZI), also known as Diemailing injection, is a traditional Chinese medicine injection of the composite plant Ixeris sonchifolia Hance (also known as Kudiezi), and has been widely used to treat coronary heart disease, angina pectoris, and cerebral infarction, but its pharmacological mechanisms remain unclear. This study is designed to explore the effects of KDZI on middle cerebral artery occlusion and reperfusion $(\mathrm{MCAO} / \mathrm{R})$ rats, and to identify metabolic features of cerebral ischemia reperfusion by using a nontargeted metabolic profiling method based on ultra-performance liquid chromatography-high resolution mass spectrometry (UPLC-HRMS). In this process, 32 potential biomarkers were found in plasma. KDZI significantly upregulated the levels of taurochenodesoxycholic acid, leucine, l-phenylalanine, l-tryptophan, arachidonic acid (ARA), and phosphatidyl ethanolamines (PE), phosphatidyl cholines (PC) and downregulated the levels of 1-valine and 5-hydroxyindole-3-acetic acid (5-HIAA) in plasma. The results indicated that the mechanisms of KDZI on MCAO/R were related to the mechanisms of amino acid and lipid metabolism.
\end{abstract}

Keywords: Kudiezi injection; UPLC-HRMS; middle cerebral artery occlusion and reperfusion

\section{Introduction}

The process of aging has hastened the increasing number of elderly people in human society. In the meantime, the risk of cerebrovascular disease increases year by year. Among cerebrovascular disease, ischemic cerebrovascular disease is one of the diseases with the highest morbidity, which has the features of high incidence, high morbidity, and high mortality [1]. Cerebral ischemic infarction can cause disability, cognitive dysfunction, vascular dementia, and even death. The increasing morbidity and mortality of cerebral ischemia seriously affects the physical and mental health of the elderly, as well as an unbearable financial toll for both society and families [2].

When ischemic cerebrovascular disease attacks, the priority goal is to recover the blood supply of the brain as soon as possible to avoid brain tissue necrosis. However, if the treatment is not timely, cerebral ischemia reperfusion injury will occur. Therefore, ischemic cerebrovascular patients should be evaluated rapidly in the early phase to receive timely and appropriate treatment. 
Unfortunately, the diagnostic features of the ischemic cerebrovascular are still limited. Typical diagnosis relies upon medical equipment, such as computed tomography (CT) or magnetic resonance imaging (MRI), but these examinations are time-consuming and costly. Therefore, it is necessary to develop novel biomarkers through blood metabolomics for the diagnosis and prognosis of ischemic cerebrovascular [3].

Till now, the mechanisms for ischemic cerebrovascular have been dramatically elucidated and considered a series of complex pathophysiological processes, including energy metabolism disorder, toxicity of excitatory amino acids, overloaded intracellular calcium, increase of oxygen free radicals, endothelial cells injury, apoptosis gene activation and inflammatory response [4-7], but the metabolite profiling of ischemic cerebrovascular disease remains unknown. Therefore, the study of the metabolic variations might be expected to provide new insights into the pathogenesis of ischemic cerebrovascular disease.

The other major challenge for ischemic cerebrovascular disease is that no effective and generally accepted medicine is available except for tissue-type plasminogen activator (t-PA ) [8]. Unfortunately, the clinical application of t-PA is limited because of the symptomatic intracerebral hemorrhage adverse reaction and narrow therapeutic window. Increasing amounts of evidence in treating ischemic cerebrovascular disease demonstrate that treatment regimens containing multiple drugs lead to maximal therapeutic efficacy with minimal adverse effects. Therefore, combination therapy becomes a rational and promising choice, and has been advocated in traditional Chinese medicine (TCM) to prevent and cure ischemic cerebrovascular disease for thousands of years. Varieties of herbal formulae or extracts have been shown to exhibit anti-cerebral ischemic effects, and Kudiezi injection (KDZI) is one of the most representative ones. KDZI, also known as Diemailing injection, is a TCM injection of the composite plant Ixeris sonchifolia Hance (also known as Kudiezi) [9]. KDZI contains flavonoids, adenosine, sesquiterpene lactones, and triterpenoid saponins, etc. [10,11]. Studies have shown that KDZI can inhibit thrombosis, increase fibrinolytic activity, dilate blood vessels, reduce vascular resistance, and increase brain and blood flow [12]. KDZI also has protective effect on brain injury in rats with cerebral ischemia reperfusion, but the mechanism is not clear yet [13-16]. Systemic studies are, therefore, required to provide a comprehensive evaluation of the efficacy of KDZI.

Metabolomics, an important part of systemic biology, was initially defined by professor Nicholson et al. [17]. Metabolomics aims at comprehensive characterization of the total metabolome in a biological system and the responses that dynamic metabolomics response to alterations of inner and external factors $[18,19]$. In recent years, metabolomics has been widely used in the diagnosis of heart disease [20], diabetes mellitus [21], and cancer [22]. However, the plasma metabolic profiling of ischemic cerebrovascular remains poorly understood and no metabolomic assessment of the anti-cerebral ischemia effects of KDZI is published.

In this study, a middle cerebral artery occlusion reperfusion (MCAO/R) rat model was established to mimic ischemic cerebrovascular, and plasma samples were collected from the MCAO/R rat model, controls, sham surgery group, and KDZI-treated group, an ultra-performance liquid chromatography-high resolution mass spectrometry (UPLC-HRMS)-based metabolomics approach was developed to characterize the metabolic biomarkers on ischemic cerebrovascular, and to discover potential targets of KDZI on ischemic cerebrovascular treatment. Partial least squares-discriminant analysis (PLS-DA) was further utilized to identify the ischemic cerebrovascular-related metabolic alterations and investigate the anti-cerebral ischemia mechanisms of KDZI. In addition, the study provided a reasonable basis for clinical treatment programs and a scientific example to highlight the power of the LC-HRMS system for sophisticated biological datasets. 


\section{Results}

\subsection{Effects of KDZI on Pathological Brain Morphology Alterations in MCAO/R Rats}

Hematoxylin and eosin (HE) staining (Figure 1A) showed that no neuronal damage or inflammatory cell infiltration was observed in the cerebral cortex of rats in Sham group, the number and morphology of neuronal cells were normal, and the cell membrane and nucleolus were clear. However, the ischemic cortex of the brains in $\mathrm{MCAO} / \mathrm{R}$ rats displayed cytoclasis, karyopyknosis, and neuronal cells were reduced. After seven days of KDZI administration, brain edema was significantly alleviated, neuronal cells almost returned to normal, and the structure became clear and integral.

\subsection{Effects of KDZI on Infarct Size in $M C A O / R$ Model Rats}

As shown in Figure 1B,C, the results of 2,3,5-triphenyltetrazolium chloride (TTC) staining demonstrated that the relative area ratio of the infarcted area of rats in the model group was 0.232 , which was statistically significant (compared with rats in the sham group, $p<0.01$ ), indicating that the $\mathrm{MCAO} / \mathrm{R}$ model was successfully established. Compared with the $\mathrm{MCAO} / \mathrm{R}$ group, the relative ratio of the infarcted area of rats in the KDZI administration group was 0.108 , indicating that the KDZI significantly reduced the infarct size. The results indicated that KDZI can significantly reduce the cerebral infarction size and ameliorate cerebral ischemia.

A

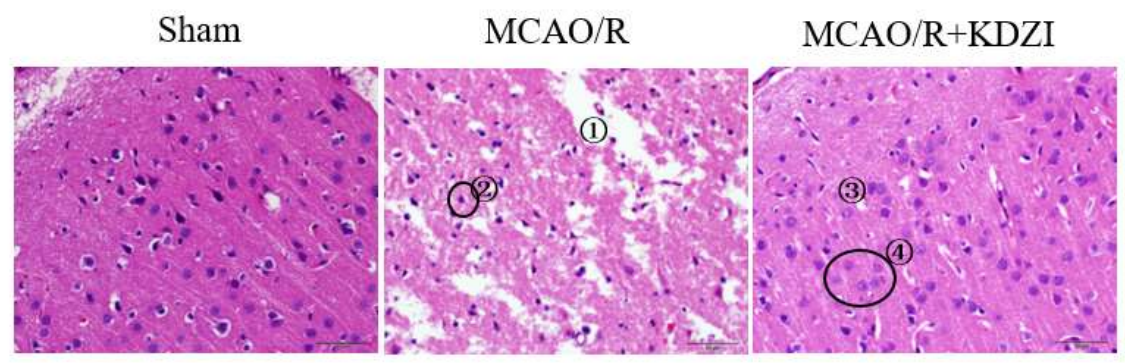

B

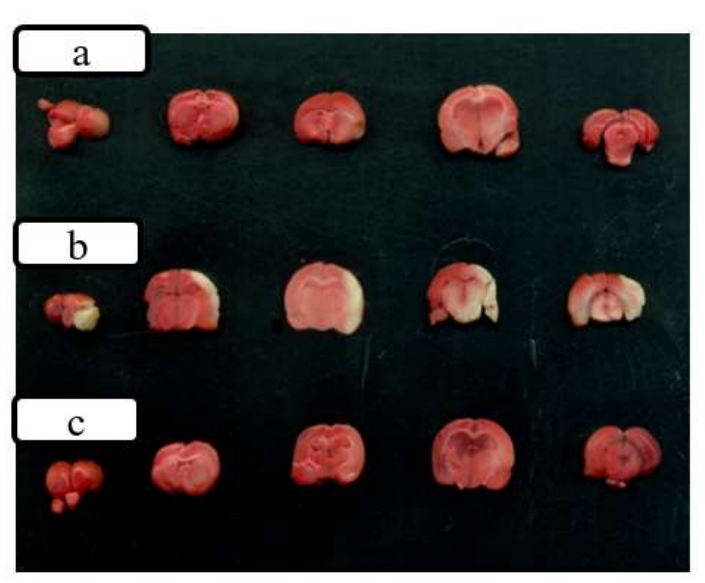

C

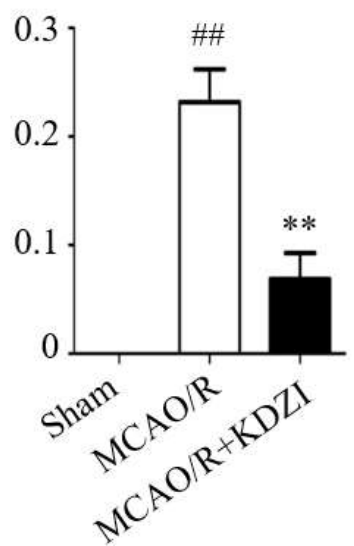

Figure 1. The representative images of $\operatorname{HE}(\mathbf{A})$ and TTC $(\mathbf{B}, \mathbf{C})$ stainings of brain sections showing the neuroprotective effect of KDZI on focal cerebral ischemia. The place marked by (1) in Figure 1A indicates cytoclasis and (2) indicates the karyopyknosis of the cortex of the brains in MCAO/R rats. (3) indicates brain edema was significantly alleviated and (4) indicates the neuronal cells almost returned to normal. Figure 1B shows: (a) the sham group; (b) MCAO/R group; and (c) MCAO/R + KDZI group. Data are presented as mean \pm SD. ${ }^{\# \#} p<0.05$ with the sham group, ${ }^{* *} p<0.05$ compared with $\mathrm{MCAO} / \mathrm{R}$ group. 


\subsection{Metabolic Profiling of Plasma Sample}

To analyze the profile of the metabolites, the signal responses of plasma metabolites in $\mathrm{ESI}^{+}$and $\mathrm{ESI}^{-}$modes were combined. Both $\mathrm{ESI}^{+}$and $\mathrm{ESI}^{-}$displayed obvious differences in the metabolite profile in the plasma samples, indicating that the combination of positive and negative modes can be used to analyze plasma metabolism to a certain degree.

The quality control (QC) samples were used in the optimization of separation and determination conditions. The typical base peak intensity chromatograms of plasma samples, obtained in ESI ${ }^{+}$and ESI $^{-}$under the optimized conditions, are presented in Figure 2. Some distinctly different peaks between the sham group and the ischemia model group were found in the base peak intensity chromatograms and were then highlighted. The precision and repeatability of the system were evaluated by the reduplicate analysis of six injections of the same QC sample. The relative standard deviation (RSD) of retention times $\left(t_{R}\right)$ and peak intensities for the selected ions from QC samples were calculated to evaluate the method performance.

As shown in Table 1, the RSD of the retention times for precision, stability and repeatability were $0.035-0.25 \%, 0.09-1.88 \%$, and $0.04 \sim 0.59 \%$ in positive ion mode and $0.03-0.65 \%, 0.07-0.56 \%$, and $0.08-0.56 \%$ in negative ion mode; the RSD of the peak intensities for precision, stability, and repeatability were $3.41-7.10 \%, 3.72-7.70 \%$, and $4.52-6.98 \%$ in positive ion mode and $2.91-7.33 \%$, $2.30-7.07 \%$, and $3.45-8.33 \%$ in negative ion mode. The results of the precision, stability, and repeatability demonstrates that the proposed method is a robust method and the analysis in the study is satisfied.

A

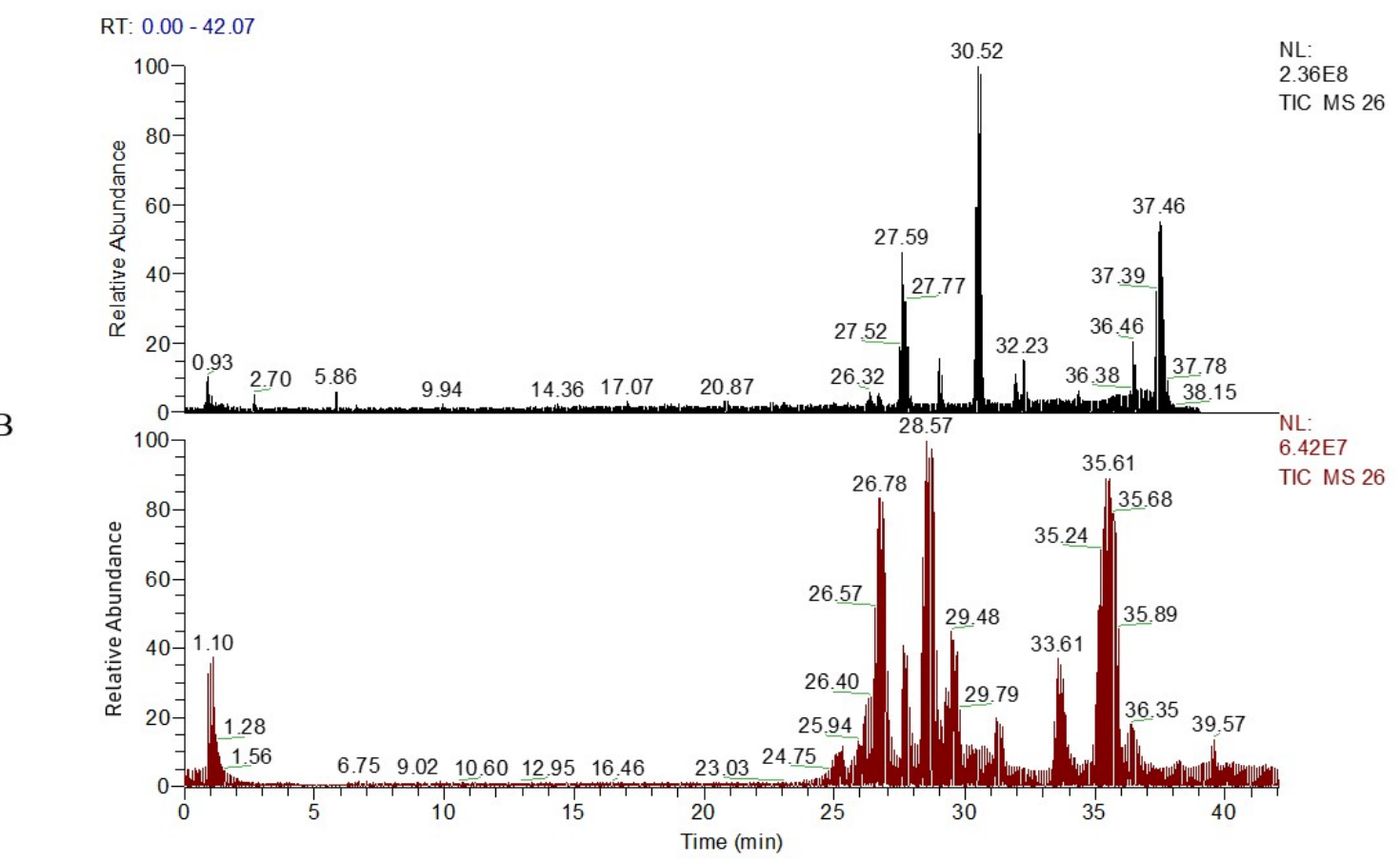

Figure 2. Representative UPLC-HR-MS total ion chromatograms (TICs) of serum samples from ESI ${ }^{+}$ model $(\mathbf{A})$, intensity of TIC $=2.36 \times 10^{8} ;$ and $\mathrm{ESI}^{-}$model $(\mathbf{B})$, intensity of $\mathrm{TIC}=6.42 \times 10^{7}$. 
Table 1. The analytical performance of the UPLC-MS method $(n=6)$.

\begin{tabular}{|c|c|c|c|c|c|c|}
\hline \multirow{2}{*}{$t_{R}-m / z$} & \multicolumn{2}{|c|}{ Precision $(R S D, \%)$} & \multicolumn{2}{|c|}{ Stability $(R S D, \%)$} & \multicolumn{2}{|c|}{ Repeatability $(R S D, \%)$} \\
\hline & $t_{R}$ & Peak Intensity & $\mathbf{t}_{\mathbf{R}}$ & Peak Intensity & $t_{R}$ & Peak Intensity \\
\hline \multicolumn{7}{|c|}{ Positive } \\
\hline $2.99-453.34$ & 0.25 & 3.41 & 1.88 & 7.70 & 0.59 & 6.51 \\
\hline $5.38-215.01$ & 0.08 & 7.10 & 0.33 & 7.28 & 0.20 & 6.98 \\
\hline $9.89-319.23$ & 0.17 & 5.80 & 0.13 & 3.72 & 0.17 & 6.51 \\
\hline $14.38-582.38$ & 0.14 & 5.35 & 0.23 & 6.22 & 0.28 & 4.52 \\
\hline $17.44-255.23$ & 0.035 & 5.14 & 0.09 & 6.89 & 0.04 & 5.11 \\
\hline \multicolumn{7}{|c|}{ Negative } \\
\hline $0.79-269.0$ & 0.65 & 7.49 & 0.56 & 7.07 & 0.56 & 6.83 \\
\hline $11.33-544.34$ & 0.09 & 4.99 & 0.07 & 4.23 & 0.09 & 4.99 \\
\hline $12.06-478.34$ & 0.15 & 2.91 & 0.14 & 2.30 & 0.15 & 3.45 \\
\hline $14.63-341.32$ & 0.11 & 7.33 & 0.12 & 6.31 & 0.11 & 8.33 \\
\hline $19.42-654.33$ & 0.03 & 4.94 & 0.08 & 5.91 & 0.08 & 6.44 \\
\hline
\end{tabular}

Note: $t_{R}$ : Retention time.

\subsection{Multivariate Data Analysis of UPLC-LTQ/Orbitrap Data}

TTC and HE stainings showed that the injury of cerebral ischemia-reperfusion of rats with treatment of KDZI was significantly improved compared with that of the MCAO/R group, so we used non-targeted LC-MS to detect the anti-ischemia mechanism of KDZI. Firstly, based on the ANOVA test, we found a total of 449 ions changed significantly $(p<0.05)$ between MCAO/R and sham groups. The ions with statistical significance were merged and introduced into SIMCA-P for multivariate analyses. According to the PCA model shown in Figure 3A,E, there was a good separation among sham, $\mathrm{MCAO} / \mathrm{R}$, and KDZI groups, the R2X(cum) value were 0.71 in $\mathrm{ESI}^{+}$and 0.647 in $\mathrm{ESI}^{-}$mode, respectively, which indicated that acute ischemia reperfusion and the intervention of KDZI can cause significant changes in the related components.

In order to examine the possible influence on the metabolic pattern of KDZI, and to verify the metabolic features of MCAO/R, the supervised method PLS-DA was used to isolate the variables that were responsible for differences among three groups. In ESI ${ }^{+}$mode, $\mathrm{R} 2 \mathrm{X}$ (cum) $=0.797, \mathrm{R} 2 \mathrm{Y}$ (cum) $=0.989$; in ESI ${ }^{-}$mode, R2X(cum) $=0.657$, and R2Y (cum) $=0.949$ (Figure 3B,F). The loading S-plot shown in Figure 3C,G explains the separation above and is used to discover biomarkers between the three groups. Furthermore, the robustness of the PLS-DA models was assessed by a 200-times permutation test. No over-fitting $(\mathrm{Q} 2 \mathrm{Y}>0.5)$ was observed according to the results of the permutation test (Figure 3D,H) [23]. 
A

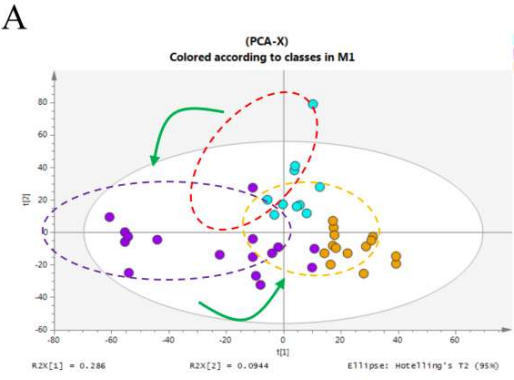

$\mathrm{C}$

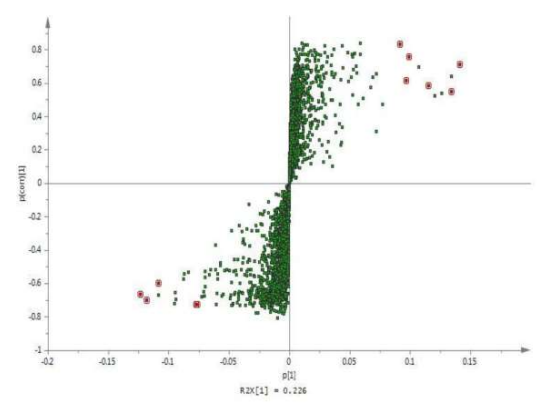

B

$$
0.2
$$

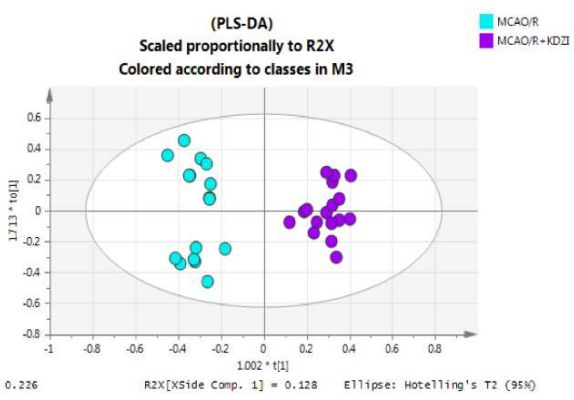

$\mathrm{D}$

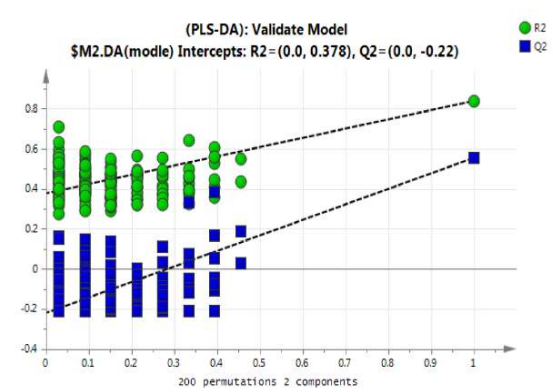

$\mathrm{E}$

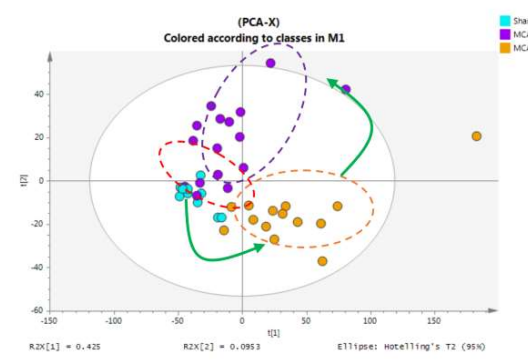

G

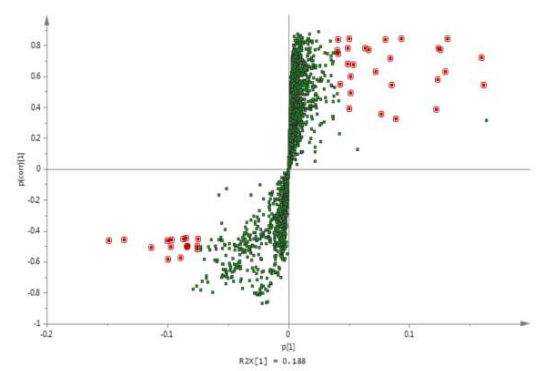

$\mathrm{F}$

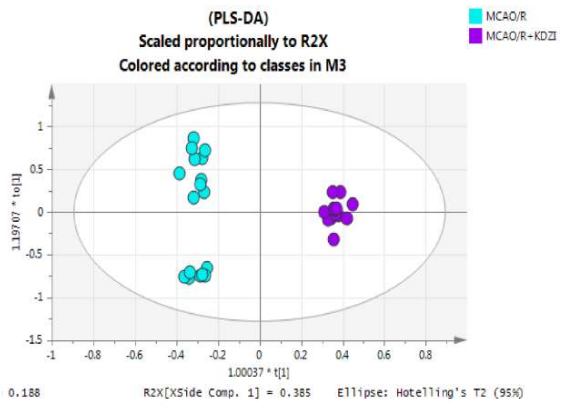

$\mathrm{H}$

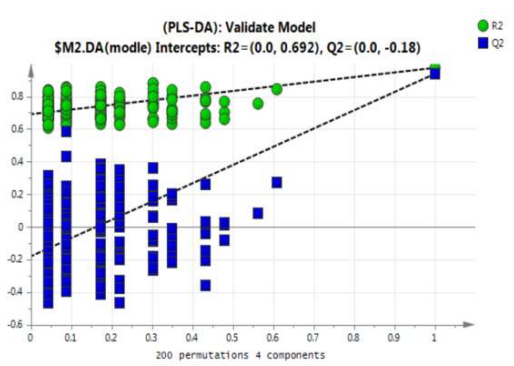

Figure 3. Multivariate data analysis of LC-MS in plasma obtained from sham, MCAO/R, and MCAO/R + KDZI group rats. $(\mathrm{A}, \mathrm{E})$ are principal component analysis (PCA) score plots for the components indicating the separation among the three groups; $(\mathbf{B}, \mathbf{F})$ are score scatter plots generated from partial least squares discriminate analysis (PLS-DA); (C,G) are S-plot of PLS-DA explaining the separation of $(\mathbf{A}, \mathrm{E})$ (variable importance in the projection(VIP) $>1.0$ have been marked); and $(\mathbf{D}, \mathbf{H})$ are the statistical validation of the PLS-DA model by permutation testing. (A-D) were under positive ion mode; and (E-H) were under negative ions mode.

\subsection{Identification of Metabolites}

As illustrated by PLS-DA plot, 210 ions were selected (VIP> 1) for chemical structure identification, which contributed to the good separation of sham, MCAO/R, and KDZI rats. Based on the published 
strategy [24,25], the following steps were performed for the identification of chemical structures in this study. The quasi-molecular ions were confirmed and the exact masses of the monoisotopic molecular weights were used to search reliable online databases such as the Human Metabolome Database [26] and Metlin [27]. The MS/MS spectra were analyzed to confirm the structure of the identified metabolites by the Mass Bank [28] and Lipidbank [29]. Identification of MS/MS using the mzCloud database, and the structures of the compounds were identified through manual comparison. Compound Discoverer 2.0 software (ThermoFisher Scientific Inc, USA) matches the mzCloud spectra of $\mathrm{MS}^{1}$ and $\mathrm{MS}^{2}$ mass spectrum data with accurate molecular weight. The parameters are MS ${ }^{1}$ mass accuracy $<5$ ppm and the library search algorithm is HighChem Low + High Res. The score matching threshold is greater than, or equal to, 60 (the higher the matching is, the more accurate it is 100). During the search process, isotope matching and deduction of background noise ( $\mathrm{S} / \mathrm{N}$ ratio $<10,000$ is cutoff) are performed.

Subsequently, 32 metabolites were identified. The names of the biomarkers, the related enzymes and the related metabolic pathways are presented in Table 2. Among them, the levels of taurochenodesoxycholic acid, leucine, 1-phenylalanine, 1-tryptophan, arachidonic acid, and PE were significantly decreased in the MCAO/R group, compared with the sham group, after seven days of KDZI administration, the compounds mentioned above were increased. In contrast, the level of l-valine and 5-hydroxyindole-3-acetic acid (5-HIAA) in the plasma of the MCAO/R group were increased, and KDZI decreased the contents of L-valine and 5-HIAA in plasma $(p<0.05)$.

Table 2. Potential plasma biomarkers detected by LC-MS and their intensities in the sham, MCAO/R, and MCAO/R + KDZI groups analyzed by one-way ANOVA.

\begin{tabular}{|c|c|c|c|c|c|c|c|}
\hline \multirow{2}{*}{ No. } & \multirow{2}{*}{$t_{R}$} & \multirow{2}{*}{$m / z$} & \multirow{2}{*}{ Formula } & \multirow{2}{*}{ Identification } & \multirow{2}{*}{$\begin{array}{l}\text { Adduct } \\
\text { Type }\end{array}$} & \multicolumn{2}{|c|}{ Flod Change } \\
\hline & & & & & & $\begin{array}{l}\text { MCAO/R/ } \\
\text { Sham }\end{array}$ & $\begin{array}{l}\mathrm{MCAO} / \mathrm{R}+ \\
\mathrm{KDZI} / \mathrm{MCAO} / \mathrm{R}\end{array}$ \\
\hline 1 & 0.93 & 118.0856 & $\mathrm{C}_{5} \mathrm{H}_{11} \mathrm{NO}_{2}$ & L-Valine & $\mathrm{M}+\mathrm{H}$ & 1.40 & 0.65 \\
\hline 2 & 1.617 & 132.1013 & $\mathrm{C}_{6} \mathrm{H}_{13} \mathrm{NO}_{2}$ & Isoleucine & $\mathrm{M}+\mathrm{H}$ & 0.68 & 1.47 \\
\hline 3 & 2.829 & 166.0856 & $\mathrm{C}_{9} \mathrm{H}_{11} \mathrm{NO}_{2}$ & L-Phenylalanine & $\mathrm{M}+\mathrm{H}$ & 0.66 & 1.45 \\
\hline 4 & 5.51 & 205.0965 & $\mathrm{C}_{11} \mathrm{H}_{12} \mathrm{~N}_{2} \mathrm{O}_{2}$ & L-Tryptophan & $\mathrm{M}+\mathrm{H}$ & 0.81 & 1.36 \\
\hline 5 & 1.89 & 192.0655 & $\mathrm{C}_{10} \mathrm{H}_{9} \mathrm{NO}_{3}$ & 5-Hydroxyindole-3-acetic acid & $\mathrm{M}+\mathrm{H}$ & 1.34 & 0.64 \\
\hline 6 & 8.585 & 132.1013 & $\mathrm{C}_{6} \mathrm{H}_{13} \mathrm{NO}_{2}$ & Leucine & $\mathrm{M}+\mathrm{H}$ & 0.68 & 1.44 \\
\hline 7 & 28.55 & 480.28 & $\mathrm{C}_{26} \mathrm{H}_{45} \mathrm{NO}_{6} \mathrm{~S}$ & Taurochenodesoxycholic acid & $\mathrm{M}-\mathrm{H}_{2} \mathrm{O}-\mathrm{H}$ & 0.71 & 1.43 \\
\hline 8 & 143 & 283.2623 & $\mathrm{C}_{18} \mathrm{H}_{34} \mathrm{O}_{2}$ & Oleic acid & $\mathrm{M}-\mathrm{H}$ & 0.78 & 1.56 \\
\hline 9 & 33.932 & 305.2468 & $\mathrm{C}_{20} \mathrm{H}_{32} \mathrm{O}_{2}$ & Arachidonic acid & $\mathrm{M}+\mathrm{H}$ & 0.63 & 1.27 \\
\hline 10 & 35.14 & 283.2623 & $\mathrm{C}_{18} \mathrm{H}_{36} \mathrm{O}_{2}$ & Stearic acid & $\mathrm{M}-\mathrm{H}$ & 0.71 & 1.39 \\
\hline 11 & 23.119 & 468.3067 & $\mathrm{C}_{22} \mathrm{H}_{46} \mathrm{NO}_{7} \mathrm{P}$ & PE $(17: 0 / 0: 0)$ & $\mathrm{M}+\mathrm{H}$ & 0.61 & 1.38 \\
\hline 12 & 26.884 & 482.3222 & $\mathrm{C}_{23} \mathrm{H}_{48} \mathrm{NO}_{7} \mathrm{P}$ & LysoPE (0:0/18:0) & $\mathrm{M}+\mathrm{H}$ & 0.81 & 1.34 \\
\hline 13 & 37.372 & 482.3221 & $\mathrm{C}_{23} \mathrm{H}_{48} \mathrm{NO}_{7} \mathrm{P}$ & LysoPE (18:0/0:0) & $\mathrm{M}+\mathrm{H}$ & 0.51 & 1.31 \\
\hline 14 & 25.379 & 482.3225 & $\mathrm{C}_{23} \mathrm{H}_{48} \mathrm{NO}_{7} \mathrm{P}$ & LysoPC (15:0) & $\mathrm{M}+\mathrm{H}$ & 0.61 & 1.20 \\
\hline 15 & 29.152 & 496.3376 & $\mathrm{C}_{24} \mathrm{H}_{50} \mathrm{NO}_{7} \mathrm{P}$ & LysoPC (16:0) & $\mathrm{M}+\mathrm{H}$ & 0.78 & 1.45 \\
\hline 16 & 33.86 & 508.3738 & $\mathrm{C}_{26} \mathrm{H}_{54} \mathrm{NO}_{6} \mathrm{P}$ & LysoPC (p-18:0) & $\mathrm{M}+\mathrm{H}$ & 0.81 & 1.29 \\
\hline 17 & 34.499 & 510.3526 & $\mathrm{C}_{25} \mathrm{H}_{52} \mathrm{NO}_{7} \mathrm{P}$ & LysoPE (0:0/20:0) & $\mathrm{M}+\mathrm{H}$ & 0.74 & 1.32 \\
\hline 18 & 32.943 & 510.3526 & $\mathrm{C}_{25} \mathrm{H}_{52} \mathrm{NO}_{7} \mathrm{P}$ & LysoPC (17:0) & $\mathrm{M}+\mathrm{H}$ & 0.69 & 1.42 \\
\hline 19 & 24.784 & 518.3221 & $\mathrm{C}_{26} \mathrm{H}_{48} \mathrm{NO}_{7} \mathrm{P}$ & LysoPC (18:3(6z, 9z, 12z)) & $\mathrm{M}+\mathrm{H}$ & 0.89 & 1.23 \\
\hline 20 & 29.081 & 518.32 & $\mathrm{C}_{26} \mathrm{H}_{48} \mathrm{NO}_{7} \mathrm{P}$ & PC $(18: 3(9 z, 12 z, 15 z) / 0: 0)$ & $\mathrm{M}+\mathrm{H}$ & 0.82 & 1.42 \\
\hline 21 & 31.039 & 522.3535 & $\mathrm{C}_{26} \mathrm{H}_{52} \mathrm{NO}_{7} \mathrm{P}$ & LysoPC $(18: 1(9 \mathrm{z}))$ & $\mathrm{M}+\mathrm{H}$ & 0.71 & 1.28 \\
\hline 22 & 33.776 & 522.3535 & $\mathrm{C}_{26} \mathrm{H}_{52} \mathrm{NO}_{7} \mathrm{P}$ & LysoPC (18:1(11z)) & $\mathrm{M}+\mathrm{H}$ & 0.63 & 1.32 \\
\hline 23 & 15.814 & 536.333 & $\mathrm{C}_{27} \mathrm{H}_{54} \mathrm{NO}_{7} \mathrm{P}$ & LysoPE (0:0/22:1(13z)) & $\mathrm{M}+\mathrm{H}$ & 0.89 & 1.41 \\
\hline 24 & 17.288 & 536.3331 & $\mathrm{C}_{27} \mathrm{H}_{54} \mathrm{NO}_{7} \mathrm{P}$ & LysoPE (22:1(13z)/0:0) & $\mathrm{M}+\mathrm{H}$ & 0.71 & 1.24 \\
\hline 25 & 35.514 & 536.3695 & $\mathrm{C}_{27} \mathrm{H}_{54} \mathrm{NO}_{7} \mathrm{P}$ & LysoPE (0:0/22:1(13Z)) & $\mathrm{M}+\mathrm{H}$ & 0.83 & 1.42 \\
\hline 26 & 32.715 & 538.3848 & $\mathrm{C}_{27} \mathrm{H}_{56} \mathrm{NO}_{7} \mathrm{P}$ & LysoPE (0:0/22:0) & $\mathrm{M}+\mathrm{H}$ & 0.79 & 1.23 \\
\hline 27 & 30.365 & 538.3858 & $\mathrm{C}_{27} \mathrm{H}_{56} \mathrm{NO}_{7} \mathrm{P}$ & LysoPE $(22: 0 / 0: 0)$ & $\mathrm{M}+\mathrm{H}$ & 0.70 & 1.32 \\
\hline 28 & 24.365 & 542.3221 & $\mathrm{C}_{28} \mathrm{H}_{48} \mathrm{NO}_{7} \mathrm{P}$ & LysoPC $(20: 5(5 z, 8 z, 11 z, 14 z, 17 z))$ & $\mathrm{M}+\mathrm{H}$ & 0.61 & 1.33 \\
\hline 29 & 30.525 & 546.3532 & $\mathrm{C}_{28} \mathrm{H}_{52} \mathrm{NO}_{7} \mathrm{P}$ & LysoPC $(20: 3(5 z, 8 z, 11 z))$ & $\mathrm{M}+\mathrm{H}$ & 0.74 & 1.34 \\
\hline 30 & 29.248 & 546.3534 & $\mathrm{C}_{28} \mathrm{H}_{52} \mathrm{NO}_{7} \mathrm{P}$ & LysoPC (20:3(8z, 11z, 14z)) & $\mathrm{M}+\mathrm{H}$ & 0.82 & 1.31 \\
\hline 31 & 34.412 & 548.369 & $\mathrm{C}_{28} \mathrm{H}_{54} \mathrm{NO}_{7} \mathrm{P}$ & LysoPC $(20: 2(11 z, 14 z))$ & $\mathrm{M}+\mathrm{H}$ & 0.82 & 1.34 \\
\hline 32 & 29.521 & 570.3532 & $\mathrm{C}_{30} \mathrm{H}_{52} \mathrm{NO}_{7} \mathrm{P}$ & LysoPC $(22: 5(7 z, 10 z, 13 z, 16 z, 19 z))$ & $\mathrm{M}+\mathrm{H}$ & 0.81 & 1.34 \\
\hline
\end{tabular}

Note: $t_{R}$ : Retention time. 
In addition, the hierarchical cluster analysis was performed to assess the relatedness and the distance of samples in different groups, and the heatmap was presented as a visual aid by using those biomarkers as variables. On the y-axis, the cluster was broken into three parts, including the sham group, MCAO/R group, and MCAO + KDZI (Figure 4).

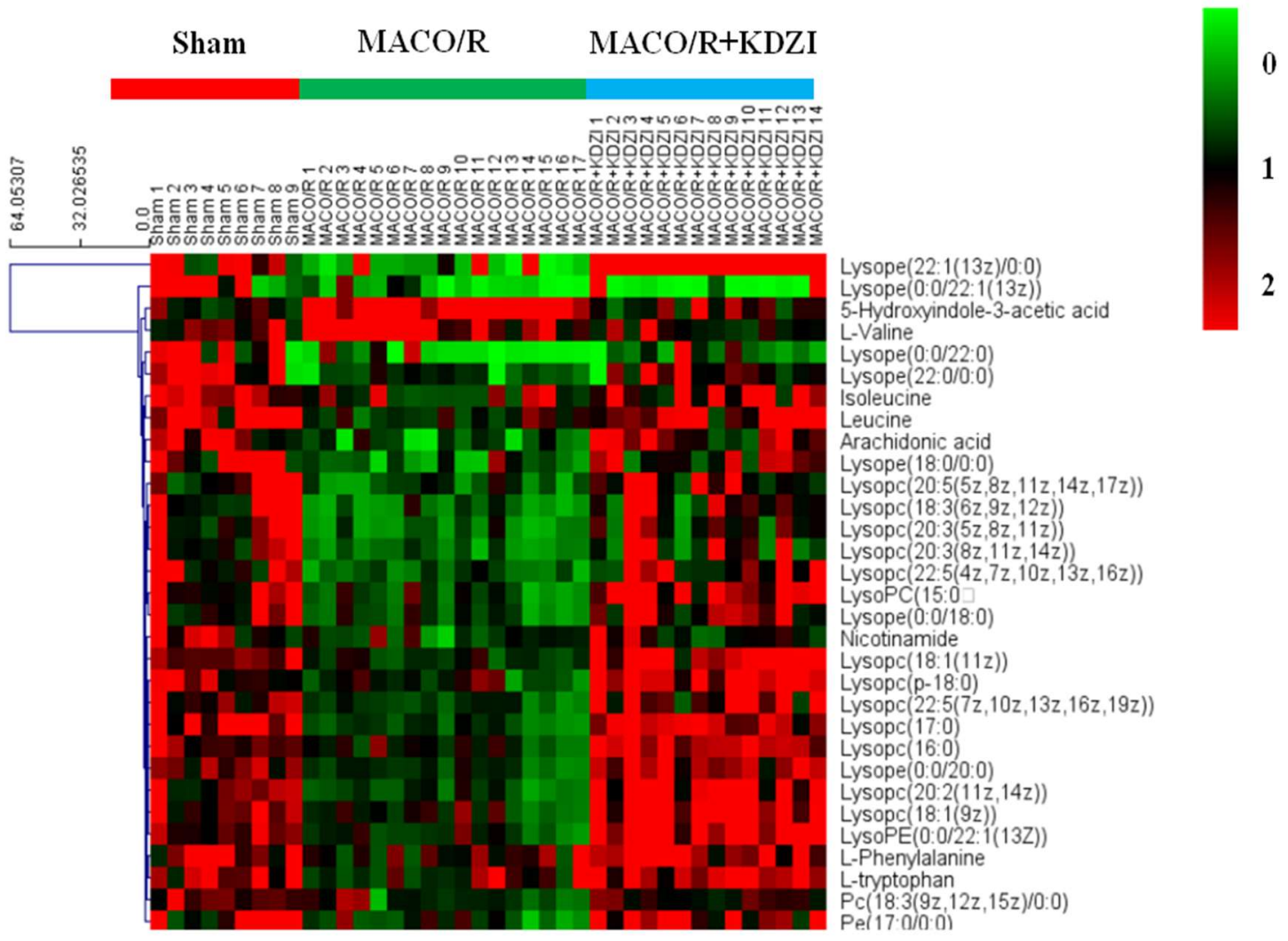

Figure 4. Heatmap of the 32 identified metabolites showing a significant difference among samples from the sham, $\mathrm{MCAO} / \mathrm{R}$, and $\mathrm{MCAO} / \mathrm{R}+\mathrm{KDZI}$ groups constructed by using by using the software Multi Experiment Viewer (MeV) v4.8. Rows: potential biomarkers; columns: samples. The color key indicates the relative amounts of metabolites: Light green represents the lowest; light red represents the highest.

\subsection{Structure Identification}

In this study, a total of 32 compounds were identified in rats' plasma. L-phenylalanine was used as an example to elucidate its structure. In HESI positive mode, a $[\mathrm{M}+\mathrm{H}]^{+}$peak could be observed, the retention time of L-phenylalanine was $2.829 \mathrm{~min}$, and the $\mathrm{m} / \mathrm{z}$ was 166.08665 (mass error $-2.4 \mathrm{ppm}$ ). Fragment ions of $m / z$ 148.92, $m / z$ 130.92, $m / z$ 119.92, and $m / z 117$ appeared in MS ${ }^{2}$ by LTQ-Orbitrap mass. The peak of $m / z 119.92$ appeared in the form of base peak in the MS ${ }^{2}$ spectrum, which differed from the peak of the excimer ion by $46 \mathrm{Da}$ and one molecule of formic acid, so it was presumed to be a carboxylic acid compound. In addition, both the excimer ions and the main fragments were even numbers. The compound is presumed to be a nitrogen-containing compound. Additionally, $m / z 148.92$ was a fragment ion of an excimer ion-missing $\mathrm{NH}_{2}$, which was identified as phenylalanine by comparison with databases, such as Metlin and HMDB. The mass spectrogram and the cleavage behavior are shown in Figure 5A,B. The identifications of other compounds are the same as above. 


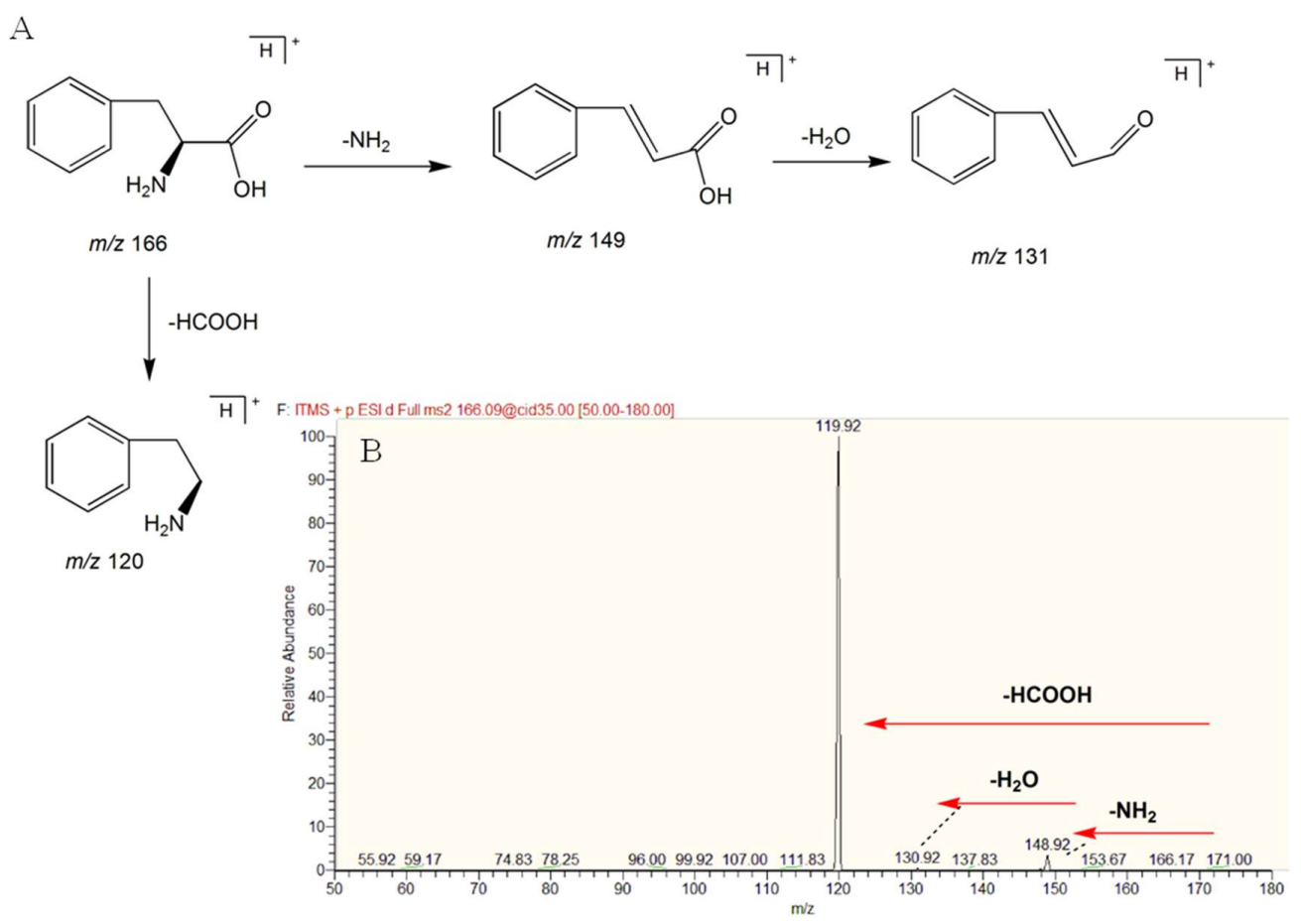

Figure 5. (A) Fragmentation pathway and product ion spectrum of L-phenylalanine; (B) MS/MS spectrum of L-phenylalanine by mzCloud.

\subsection{Metabolic Pathway Analysis}

On the basis of the identified compounds and the Kyoto Encyclopedia of Genes and Genomes online database of metabolic pathways [30], a map of metabolic pathways of the treatment of cerebral ischemia-reperfusion injury with KDZI was constructed, shown in Figure 6 and Table 3. The relevant metabolic pathways and corresponding $p$-values and FDR correction (FDR correction value of the $p$-value) are shown in Table 3 and Figure 6. Among them, tryptophan metabolism, valine, leucine, and isoleucine degradation, and valine, leucine, and isoleucine biosynthesis were the major relevant metabolic pathways. Tryptophan, valine, leucine, and isoleucine were important factors related to cerebral ischemia.

Table 3. The information of the relevant metabolic pathways.

\begin{tabular}{lccc}
\hline \multicolumn{1}{c}{ Pathyway Name } & In Set & $p$-Value $\left(\times \mathbf{1 0}^{\mathbf{3}}\right)$ & FDR Correction $\left(\times \mathbf{1 0}^{\mathbf{3}}\right)$ \\
\hline Tryptophan metabolism & 2 & 0 & 0 \\
Fatty acid elongation unsaturated & 3 & 0.00000296 & 0.0000266 \\
Biosynthesis of unsaturated fatty acids & 3 & 0.00000723 & 0.000803 \\
Valine, leucine and isoleucine degradation & 3 & 0.0000744 & 0.00156 \\
Valine, leucine and isoleucine biosynthesis & 3 & 0.000422 & 0.00814 \\
Aminoacyl-tRNA biosynthesis & 4 & 0.00119 & 0.044 \\
Biosynthesis of secondary metabolites & 5 & 0.00157 & 0.0173 \\
Glucosinolate biosynthesis & 5 & 0.0332 & 0.38 \\
Metabolic pathways & 3 & 0.116 & 0.522 \\
jasmonoyl-amino acid conjugates biosynthesis I & 4 & 3.29 & 7.4 \\
jasmonoyl-amino acid conjugates biosynthesis II & 3 & 6.53 & 28.3 \\
\hline
\end{tabular}




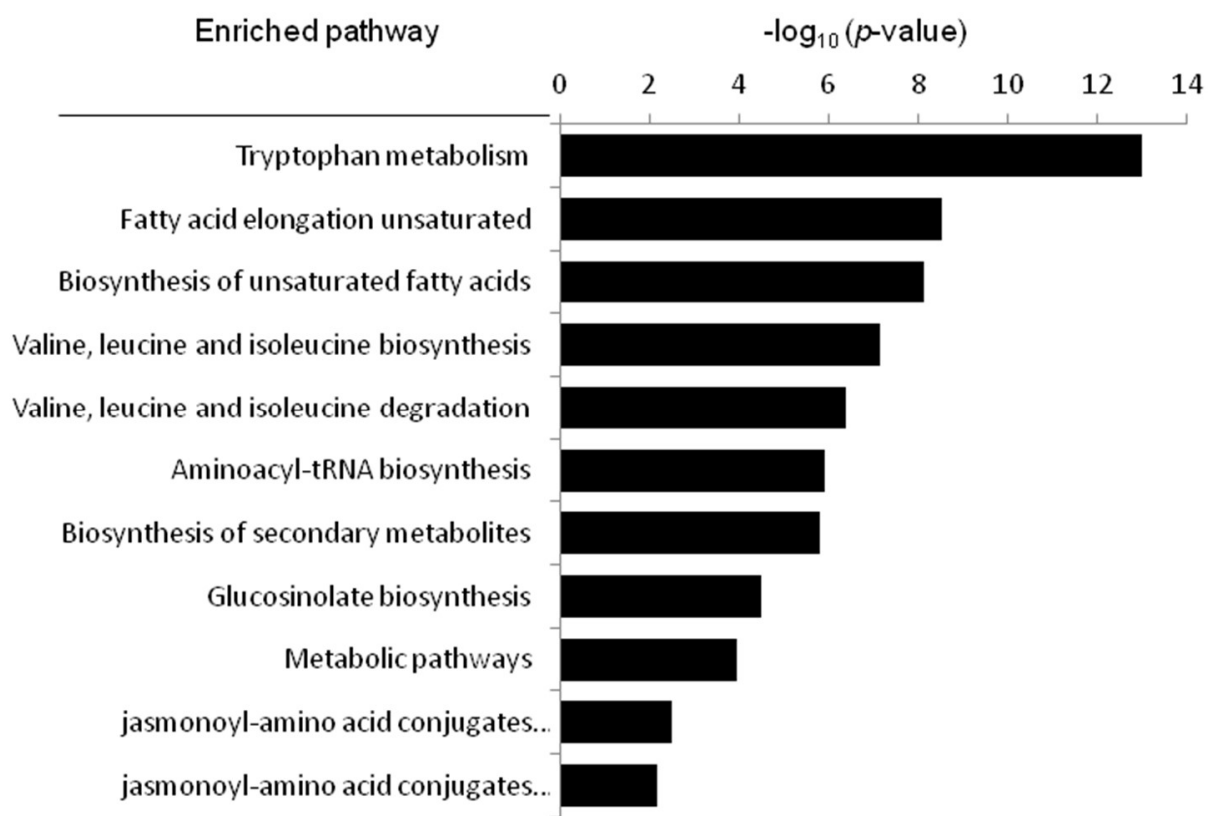

Figure 6. The metabolic pathways related to cerebral ischemia-reperfusion, as analyzed by MetaboAnalyst.

\section{Discussion}

\subsection{Characterized Potential Biomarkers}

Cerebral ischemic infarction can cause disability, cognitive dysfunction, vascular dementia and even death. The increasing morbidity and mortality of cerebral ischemia seriously affects the physical and mental health of the elderly, as well as delivering an unbearable financial toll on society and families. Therefore, it is better to find serum biomarkers as compared to physical diagnosis, such as CT, as early as possible before the ischemic injury. Hence, we designed this study to find biomarkers of cerebral ischemia reperfusion by using metabolomics technology. In this research, we found that KDZI had the effect of reducing embolism area of $\mathrm{MCAO} / \mathrm{R}$ rats and regulating of metabolites relevant to cerebral ischemia reperfusion. 32 potential biomarkers were found in plasma in the process. To date, metabolites of ischemic cerebral reperfusion rats with the treatment of Kudiezi injection have been analyzed using UHPLC-LTQ-Orbitrap, but only 15 metabolites were found in their study [31].

We observed taurochenodesoxycholic acid (TCDCA) were increased after KDZI administration to $\mathrm{MCAO} / \mathrm{R}$ rats. In the studies of excitatory neurotoxicity and hypoxia, TCDCA can maintain the function of mitochondria, decrease the calcium influx triggered by glutamate neurotoxicity, and protect nerve function. Taurine interacts with gamma-aminobutyric acid (GABA) to activate GABA receptors and glycine receptors, enhance the neuroprotective effects, assist in the transport of ions, like $\mathrm{Ca}^{2+}$, $\mathrm{Mg}^{2+}, \mathrm{Na}^{+}$, and $\mathrm{K}^{+}$, and to maintain normal osmotic pressure intracellularly and extracellularly. The decreased level of taurine indicated that brain tissues of cerebral ischemia and reperfusion rats produce excitotoxic neurotoxicity. Hua Liu et al. [32] found that taurine had the effect of an anti-free radical on cerebral ischemic reperfusion damage. Zhong Yang et al. [33]. demonstrated that TCDCA had the effects of downregulating the level of malondialdehyde (MDA) and upregulating the level of glutathione peroxidase (GSH-Px) in MCAO/R rats.

Our results showed that the level of $\mathrm{L}$-valine was decreased in $\mathrm{MCAO} / \mathrm{R}$ rats and the levels of isoleucine, L-leucine, L-phenylalanine, and L-tryptophan were increased with the treatment of KDZI. Similarly, Liu Siyi et al. [34] also found the biosynthesis of phenylalanine and tryptophan in rats with the treatment of Kudiezi injection. These amino acids enter the tricarboxylic acid cycle by deamination or transamination and promote the production of adenosinetriphosphate (ATP). Changes of these amino acid levels cause the reduction of ATP provided by tricarboxylic acid cycle. Brain cells are 
highly dependent on mitochondrial energy. Thus, the decrease of ATP provided by the tricarboxylic acid cycle can cause brain ischemia. When cerebral ischemia occurs, oxygen cannot be adequately supplied, the brain tissues of rats are mainly supplied by anaerobic glycolysis, and amino acid contents are significantly reduced. Interestingly, these amino acids can be regulated by the treatment of KDZI. As depicted in Figure 7B, the levels of isoleucine, l-leucine, 1-phenylalanine, and l-tryptophan were significantly decreased, and the level of l-valine was increased in the rats of the MCAO/R group compared with that of the sham group; with the treatment of KDZI, the levels of isoleucine, l-leucine, 1-phenylalanine, and l-tryptophan were significantly increased, and the level of l-valine was decreased.

After cerebral ischemia, a large number of free radicals are produced through arachidonic acid (ARA) metabolism and other ways, and these free radicals have neurotoxic actions on intracellular proteins, lipids, and nucleotides, resulting in neurocyte damage. The neurotoxic effects of oxygen radicals can be summarized as follows: (1) they act on polyunsaturated fatty acids, and cause lipid peroxidation; and (2) they induce the crosslink of macromolecules, such as DNA, RNA, polysaccharides, and amino acids, which result in activity reduction/loss of the large molecules. As demonstrated in Figure 7B, the level of ARA was significantly decreased in the rats of the MCAO/R group compared with that of the sham group; with the treatment of KDZI, the level of ARA was significantly increased.

Cerebral ischemia produces a large number of free radicals, and these free radicals attack nerve membranes and blood vessels that are rich in polyunsaturated fatty acids and produce lipid peroxides and hydroperoxides. The loss of the unsaturated fatty acids destruct the integrity of cellular structures. Meanwhile, the membrane permeability, ion transport, and membrane screen function are also severely affected, resulting in cell necrosis. Similarly, we found that the level of ARA in rats of MCAO/R + KDZI group increased significantly.

Twenty-two phosphatidyl ethanolamines (PE), phosphatidyl cholines (PC) were changed in $\mathrm{MCAO} / \mathrm{R}$ rats compared with the rats in Sham group. Both PE and PC were increased by treating $\mathrm{MCAO/R}$ with KDZI. Lecithin can be hydrolyzed to produce glycerol phosphorylcholine (GPC), phosphorylcholine (Pcho), and choline, the phosphorylcholine is hydrolyzed into choline with the catalysis of alkaline phosphatase; at the same time, choline produces phosphorylcholine in an ATP-containing environment and then synthesizes lecithin. The studies of Djuricic et al. [23] showed that, in the case of cerebral ischemia, the content of choline increased with the decreased of level of ATP. Kozuka et al. [35] found that, in the early stage of cerebral ischemia in spontaneous hypertensive rats, the level of choline increased at 1-2 $\mathrm{h}$. The studies showed that, three hours after cerebral ischemia-reperfusion in rats, the membrane phospholipids were largely hydrolyzed. Meanwhile, because of the decrease of ATP in the brain tissues, and the reduction of efficiency of choline synthesizing lecithin, the content of choline saw a significant increase in the left-brain tissues of MCAO/R rats, and the level of phosphorylcholine decreased.

\subsection{Possible Mechanism of Anti-Cerebral Ischaemia Reperfusion Effect KDZI}

The profile of metabolic network including the 32 significantly changed metabolites in plasma were shown in Figure 7A, B. This anti-cerebral ischemia reperfusion mechanism of KDZI can be described as below: KDZI has the effect on regulating the levels of L-valine, isoleucine, L-phenylalanine, and leucine to enter the tricarboxylic acid cycle by deamination or transamination and promote the production of ATP to provide energy for the brain and anti-cerebral ischemia. KDZI can increase ARA by regulating calcium and sodium ions and NMDARs, resulting in elevated PE, PC, and improved cerebral ischemia. On the other hand, glutamate affects 5-HT secretion, KDZI can decrease 5-HIAA secretion and improve cerebral ischemia. 
A

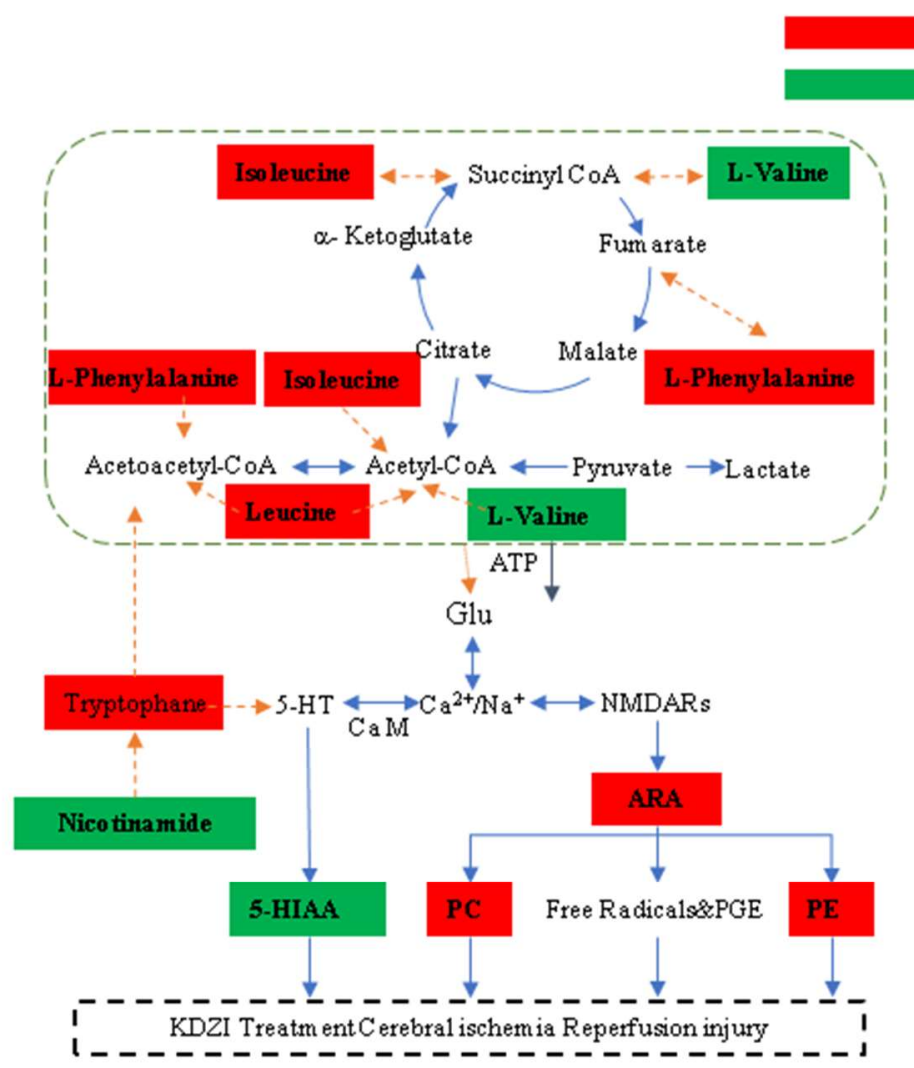

B
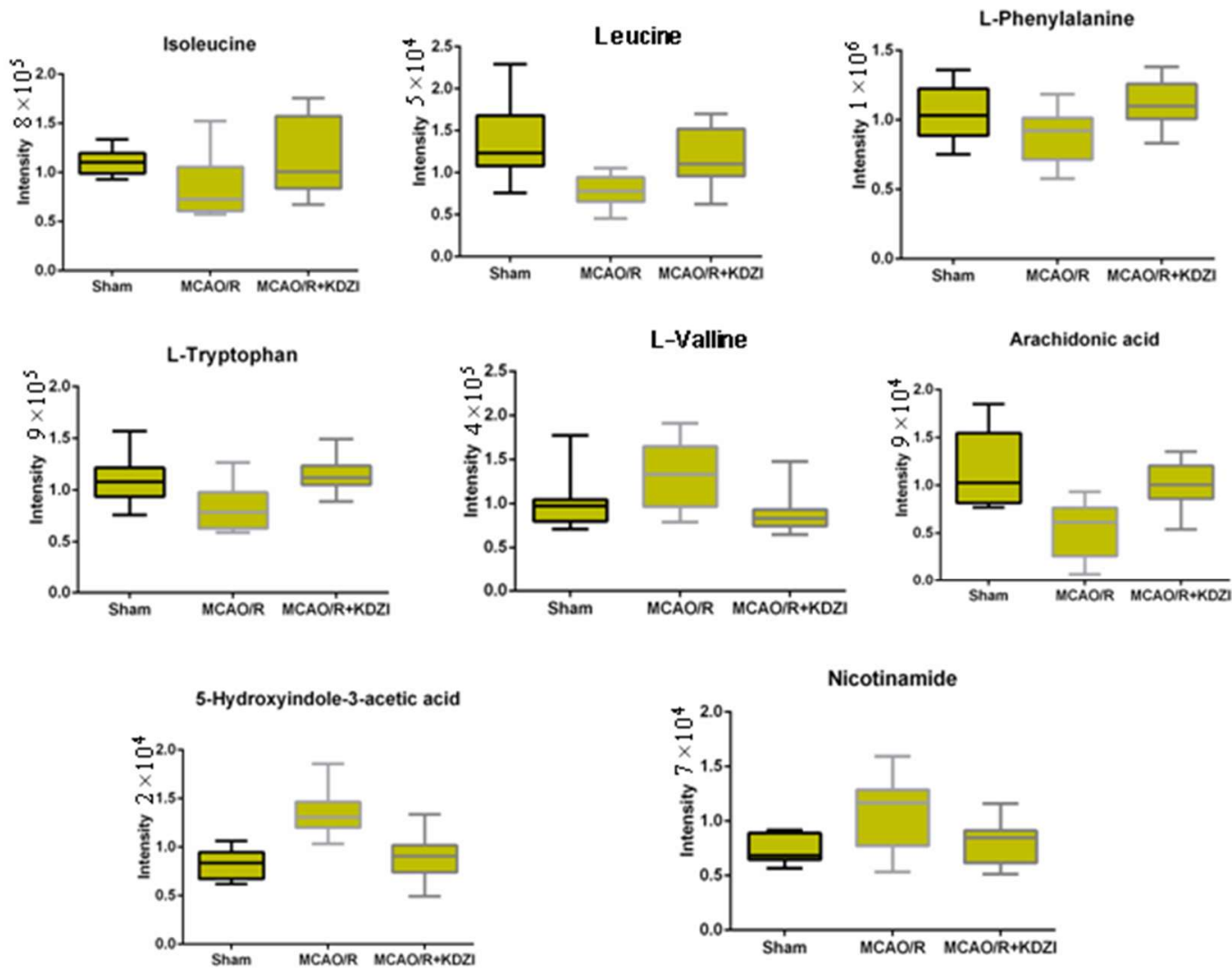

Figure 7. (A) Possible mechanism of anti-cerebral ischaemia reperfusion effect KDZI. (B) Typical metabolite variations in plasma samples from sham, MCAO/R, and MCAO/R + KDZI. Glu, glutamate; CoA, acetyl coenzyme A; ARA, arachidonate; 5-HT, 5-hydroxytryptamine; CAM, calmodulin; PE and PC, phospholipid; NMDARs, $N$-methyl-D-aspartic acid receptors. 


\section{Materials and Methods}

\subsection{Chemicals and Reagents}

Kudiezi injection (KDZI, Lot No. 140506, Jilin Tonghua Huaxia Pharmaceutical Co., Ltd., Tonghua, China), acetonitrile, methanol, and formic acid (HPLC grade) were purchased from Thermo Scientific (Waltham, MA, USA). Trypsin (mass spectrometry grade) was from Promega (Madison, WI, USA). Double distilled water $(18.2 \mathrm{M} \Omega \cdot \mathrm{cm})$ was from a Milli-Q system (Millipore, Bedford, MA, USA).

\subsection{Animals and Grouping}

Male Sprague Dawley rats (220 g $\pm 20 \mathrm{~g}$ ) were purchased from Beijing SiBeiFu Animal Technology Co. Ltd. (Beijing, China; certification number: SCXK (Jing): 2016-0002). The rats were housed in the clean level condition animal housing facilities (certification number SYXK (Jing) 2016-0038) of BUCM, at a temperature of $22 \pm 1{ }^{\circ} \mathrm{C}$, a humidity of $55 \pm 5 \%$, and a $12 \mathrm{~h}$ light/dark cycle with free access to tap water and chow. All the protocols were reviewed and approved by the Animal Care Committee of BUCM, China. The animals were allowed to acclimatize for a week while being fed with a standard diet and water ad libitum before experiments. Then they were randomly divided into three groups: sham group (SHAM), ischemic group (MCAO/R), and Kudiezi injection group (KDZI), with 15 rats in each group. In the $\mathrm{MCAO} / \mathrm{R}$ and KDZI groups, rats were subjected to $\mathrm{MCAO} / \mathrm{R}$ on the right hemisphere of the brain. The animals in the KDZI group were treated with KDZI injection $(4.2 \mathrm{~mL} / \mathrm{kg}$ i.p. The SHAM and MCAO/R groups received $0.9 \%$ sodium chloride at $4.2 \mathrm{~mL} / \mathrm{kg}$, i.p.).

\subsection{Middle Cerebral Artery Occlusion}

Rats were anesthetized with $10 \%$ chloral hydrate $(3.5 \mathrm{~mL} / \mathrm{kg}$ body weight $)$ intraperitoneally. Cerebral ischemia was conducted according to the method by Longa et al. [36,37] with some modifications. A 2-mm nylon monofilament (Guangzhou Jialing Company, Guangzhou, China) was inserted into the internal carotid artery through a small incision on the common carotid artery and advanced $18 \pm 2 \mathrm{~mm}$ to occlude the middle cerebral artery, until a slight resistance was felt. Two hours after the induction of ischemia, the filament was slowly withdrawn, and the animals were returned to their cages for a period of $2 \mathrm{~h}$ of reperfusion. Throughout the procedure, the body temperature was maintained at $37^{\circ} \mathrm{C}$, with a thermostatically-controlled infrared lamp. Rats in the sham group underwent the same procedure without monofilament insertion.

\subsection{The Stainings of TTC and HEE}

After 7 days of KDZI administration, the rats were anesthetized with $10 \%$ chloral hydrate $(3.5 \mathrm{~mL} / \mathrm{kg}$ body weight). Coronal sections of brains $(2 \mathrm{~mm})$ were immersed in $1 \%$ 2,3,5-triphenyltetrazolium chloride (TTC) in phosphate buffer saline (pH 7.4) at $37^{\circ} \mathrm{C}$ for $15 \mathrm{~min}$ and then fixed with $10 \%$ paraformaldehyde for $10 \mathrm{~min}[38,39]$. The white color represented infarct tissue and the red color represented normal tissue. TTC-stained sections were photographed and the images were analyzed using Image-Pro Plus 6.0 to calculate the infarct area. Hematoxylin and eosin (H and E) staining was conducted according to the protocol of Xiaoli Yan et al. [40]. Images were captured using an Olympus BX53 microscope (Tokyo, Japan) and analyzed using Image-Pro Plus 6.0 to calculate the IOD value.

\subsection{Plasma Pretreatment and UHPLC-LTQ/Orbitrap Analysis}

After the last administration, rats were anesthetized with $10 \%$ chloral hydrate $(3.5 \mathrm{~mL} / \mathrm{kg}$ body weight). Blood was collected from the abdominal aorta and blood samples were collected in tubes containing sodium heparin and centrifuged for $3500 \mathrm{rpm}, 10 \mathrm{~min}$ at $4{ }^{\circ} \mathrm{C}$. The plasma was removed and placed in aliquots, stored at $-80^{\circ} \mathrm{C}$ until measurement. 
Plasma sample preparation was carried out by protein precipitation with acetonitrile. Four-hundred microliters of precipitant (V (acetonitrile): V (methanol) $=3: 1$ ) was added to $200 \mu \mathrm{L}$ of plasma and the mixture was vortexed for $2 \mathrm{~min}$. After $10 \mathrm{~min}$, the mixture was centrifuged at $10,000 \mathrm{rpm}$ for $15 \mathrm{~min}$ at $4{ }^{\circ} \mathrm{C}$. Then the supernatant was transferred and dried on a nitrogen blower. Finally, the dried supernatant was re-dissolved in $100 \mu \mathrm{L}$ mobile phase (A phase $0.1 \%$ formic acid aqueous solution). To validate the analytical methodology, pooled QC samples were prepared by mixing all of the samples. Five QC samples were analyzed before sample sequencing, and during the analysis of the sample sequence, one QC sample was run after every five injections [41,42]. Metabolomics analysis was performed on a Thermo Scientific Dionex Utimate 3000 UHPLC Plus Focused coupled to a LTQ/Orbitrap MS system equipped with an electrospray ionization source operating. A $2.1 \times 100 \mathrm{~mm}$ BEH $1.7 \mu \mathrm{m}$ C18 column was equipped for all analyses. The measuring method of biomarkers was from reported research $[25,43]$. The injection volume was $2 \mu \mathrm{L}$, the flow rate was $230 \mu \mathrm{L} / \mathrm{min}$, the column temperature was $30^{\circ} \mathrm{C}$. The mobile phase was a mixture of $0.1 \%$ formic acid in water (A) and $0.1 \%$ formic acid in acetonitrile (B). Ion source temperature: $350{ }^{\circ} \mathrm{C}$, ionization source voltage: $4 \mathrm{KV}$, capillary voltage: $35 \mathrm{~V}$, tube lens voltage: $110 \mathrm{~V}$, sheath gas and auxiliary gas are high-purity nitrogen (purity $>99.99 \%$ ), sheath gas flow rate: 40 arb, the auxiliary air flow rate was 20 arb. Data were acquired using Fourier transform high resolution full sweep (TF, Full scan, Resolution 30000), MS /MS uses data-dependent acquisition, and the broken way is CID. Metabolic profiles were acquired within the range of $m / z$ 50-1500.

The Proportion of Mobile Phase B Was Optimized as Follows:

Positive ion mode: 0-4 min, 5-7\%; 4-5 min, 7-12\%; 5-6 min, 12-15\%; 6-8 min, $15-30 \%$; 8-17 min $30-48 \%$; $17-21 \mathrm{~min}, 48-49 \%$; 21-30 $\mathrm{min}, 49-59 \%$; 30-33 $\mathrm{min}, 59-61 \%$; 33-35 $\mathrm{min}, 61-80 \%$; $35-36 \mathrm{~min}$, $80-5 \%$; 36-39 $\mathrm{min}, 5-5 \%$.

Negative ion mode: $0-3 \mathrm{~min}, 3-5 \%$; 3-4 $\mathrm{min}, 5-30 \%$; 4-20 $\mathrm{min}, 30-36 \%$; $20-21 \mathrm{~min}, 36-42 \%$; 21-22 $\mathrm{min}, 42-60 \%$; 22-34 min, 60-64\%; 34-39 $\mathrm{min}, 64-80 \%$; 39-39.1 $\mathrm{min}, 80-3 \%$; 39.1-42 $\mathrm{min}, 3-3 \%$.

\subsection{Data Processing and Analysis}

The raw data analysis for plasma samples were processed using the Sieve software package version 2.1 (Thermo Fisher Scientific Inc., San Jose, CA, USA). Before chemometric analysis, the data from each sample were normalized to the sum of the peak area [44]. The pre-processed data were analyzed by principal component analysis (PCA) and quadrature signal correction partial least squares analysis (OPLS), using SIMCA-P13.0 software (Umetrics). Differential Metabolites Identification Used Compound Discoverer 2.0 for spectral matching, combined with Metlin and HMDB databases. The obtained differential components were analyzed by MeV (Multi Experiment Viewer, v4.8, TIGR) for hierarchical clustering analysis and K-mean clustering analysis. All experimental data were expressed as means $\pm S D$, and examined by one-way ANOVA test in the SPSS version 16 software package to calculate the statistical significance. $p$-values $<0.05$ were considered significant. Differences in metabolic function and pathway enrichment analysis were using KEGG (http:/ / www.genome.jp/ kegg/), MBRole (http://csbg.cnb.csic.es/mbrole2/), and Metpa (http://Metpa.metabolomics.ca), as well as other online tools.

\section{Conclusions}

In summary, plasma metabolomics analysis of LC-MS provides biomarkers for cerebral ischemia-reperfusion metabolism. Our data shows that amino acid metabolism, energy metabolism, and lipid metabolism are involved in the metabolic pathway of cerebral ischemia and reperfusion. Among them, amino acids are the most important features of cerebral ischemia-reperfusion injury, and are considered to be an important regulator to regulate cell signal transduction. The results also show that taurine and arachidonic acid can be considered as a marker for the diagnosis of clinical diagnostic markers, and LC-MS-based techniques provide a method for the analysis of cerebral ischemia-reperfusion through biological pathways. In the course of the experimental study, 
further studies are needed to examine the dynamic trends of these biomarkers and the functions of these biomarkers.

Author Contributions: Conceptualization, T.W. and B.Z.; Methodology, C.W.; Software, C.L.; Validation, C.W.; Investigation, M.W.; Resources, Q.M.; Data Curation, Y.L.; Writing-Original Draft Preparation, C.W.

Funding: This research was funded by the [National Natural Science Foundation of China (grant numbers 81773960 and 81703744 ) and was funded by the Young Teacher Project of Beijing University of Chinese Medicine (grant number 2016-JYB-JSMS-013, 2017-JYB-JS-022).

Conflicts of Interest: The authors declare no conflict of interest.

\section{References}

1. Kim, A.S.; Johnston, S.C. Claiborne Johnston. Global Variation in the Relative Burden of Stroke and Ischemic Heart Disease. Circulation 2011, 124, 314-323.

2. Pedata, F.; Dettori, I.; Coppi, E.; Melani, A.; Fusco, I.; Corradetti, R.; Pugliese, A.M. Purinergic signalling in brain ischemia. Neuropharmcaology 2016, 104, 105-130. [CrossRef] [PubMed]

3. Liu, M.; Wu, B.; Wang, W.; Lee, L.; Zhang, S.; Kong, L. Stroke in China: Epidemiology, prevention, and management strategies. Lancet Neurol. 2007, 6, 456-464. [CrossRef]

4. $\quad$ Atsushi, S.; Carolina, M.M.; Purnima, N.; Nishi, T.; Song, Y.S.; Yu, F.; Liu, J.; Lee, Y.S.; Nito, C.; Kamada, H.; et al. Oxidative stress and neuronal death/survival signaling in cerebral ischemia. Mol. Neurobiol. 2005, 31, 105-116.

5. Atsuko, K.; Luca, S. Mitochondria: From cell death executioners to regulators of cell differentiation. Trends Cell Biol. 2014, 24, 761-770.

6. Dziedzic, T. Systemic inflammation as a therapeutic target in acute ischemic stroke. Expert Rev. Neurother. 2015, 15, 523-531. [CrossRef] [PubMed]

7. Suzuki, Y.; Nagai, N.; Umemura, K. A review of the mechanisms of blood-brain barrier permeability by tissue-type plasminogen activator treatment for cerebral ischemia. Front. Cell. Neurosci. 2016, 10, 2. [CrossRef] [PubMed]

8. Li, M.; Zhou, J.; Jin, W.; Li, X.; Zhang, Y. Danhong Injection Combined With t-PA Improves Thrombolytic Therapy in Focal Embolic Stroke. Front. Pharmacol. 2018, 308, 1-11. [CrossRef] [PubMed]

9. Jiangsu Medical College. Chinese Dictionary; Shanghai Science and Technology Press: Shanghai, China, 1977.

10. Liu, R.; Zhang, X.; Wang, F.; Shang, Z.; Wang, F.; Liu, Y.; Lu, J.; Zhang, J. Rapid screening and identification of sesquiterpene lactones in Kudiezi injection based on high-performance liquid chromatography coupled with linear ion trap-orbitrap mass spectrometry. Chin. J. Natl. Med. 2018, 16, 150-160. [CrossRef]

11. Liu, X.; Tao, Y.; Wang, F.; Yao, T.; Fu, C.; Zheng, H.; Yan, Y.; Liang, X.; Jiang, X.; Zhang, Y. Kudiezi injection mitigates myocardial injury induced by acute cerebral ischemia in rats. BMC Complement. Altern. Med. 2017, 17, 8. [CrossRef] [PubMed]

12. Dai, J.; Yin, R.; Chen, X. Progress in research on the chemical constituents and pharmcaological effects of Kudie. Northwest Pharm. J. 2006, 21, 94-96.

13. Chen, C.; Jia, H.; Lv, S.; Xu, C. Protective effect of Kudiezi on acute cerebral ischemic reperfusion injury in rats. Chin. J. Clin. Pharmcaol. 2012, 28, 196-199.

14. Tan, A.; Qian, F.; Zhu, Y.; Wang, Y.; Li, X. Kudie injection on cerebral ischemia/reperfusion injury in rats. Chin. J. Hosp. Pharm. 2009, 29, 1178-1180.

15. Wang, C.X.; Liu, Y.L.; Zhao, Q. Kudizi injrction on the protective effect of cerebral ischemia. Hebei Med. 2005, $27,860-861$.

16. Chen, F.Q.; Li, Q.; Pan, C.S.; Liu, Y.Y.; Yan, L.; Sun, K.; Mao, X.W.; Mu, H.N.; Wang, M.X.; Wang, C.S.; et al. Kudiezi injection alleviates blood-brain barrier disruption after is chemia-reperfusion in rats. Microcirculation 2016, 23, 426-437. [CrossRef] [PubMed]

17. Nicholson, J.K.; Lindon, J.C.; Holmes, E. Metabonomics: Understanding the metabolic responses of living system to pathophysiological stimuli via multivariate statistical analysis of biological NMR spectroscopic data. Xenobiotica 1999, 29, 1181-1189. [CrossRef] [PubMed]

18. Nicholson, J.K.; Connelly, J.; Lindon, J.C.; Holmes, E. Metabonomics: A platform for studying drug toxicity and gene function. Nat. Rev. Drug Discov. 2002, 1, 153-161. [CrossRef] [PubMed] 
19. Lindon, J.C.; Holmes, E.; Nicholson, J.K. Metabonomics techniques and applications to pharmaceutical research \& development. Pharm. Res. 2006, 23, 1075-1088. [PubMed]

20. Brindle, J.T.; Antti, H.; Holmes, E.; Tranter, G.; Nicholson, J.K.; Bethell, H.W.; Clarke, S.; Schofield, P.M.; McKilligin, E.; Mosedale, D.E.; et al. Rapid and noninvasive diagnosis of the presence and severity of coronary heart disease using 1HNMR-based metabonomics. Nat. Med. 2002, 8, 1439-1444. [CrossRef] [PubMed]

21. Makinen, V.P.; Soininen, P.; Forsblom, C.; Parkkonen, M.; Ingman, P.; Kaski, K.; Groop, P.H.; FinnDiane Study Group; Ala-Korpela, M. 1H NMR metabonomics approach to the disease continuum of diabetic complications and premature death. Mol. Syst. Biol. 2008, 4, 167. [CrossRef] [PubMed]

22. McPhail, M.J.; Shawcross, D.L.; Lewis, M.R.; Coltart, I.; Want, E.J.; Antoniades, C.G.; Veselkov, K.; Triantafyllou, E.; Patel, V.; Pop, O.; et al. Multivariate metabotyping of plasma accurately predicts survival in patients with decompensated cirrhosis. J. Hepatol. 2016, 64, 1058-1067. [CrossRef] [PubMed]

23. Li, X.; Xu, Z.; Lu, X.; Yang, X.; Yin, P.; Kong, H.; Yu, Y.; Xu, G. Comprehensive two-dimensional gas chromatography/time-of-flight mass spectrometry for metabonomics: Biomarker discovery for diabetes mellitus. Anal. Chim. Acta 2009, 633, 257-262. [CrossRef] [PubMed]

24. Want, E.J.; Masson, P.; Michopoulos, F.; Wilson, I.D.; Theodoridis, G.; Plumb, R.S.; Shockcor, J.; Loftus, N.; Holmes, E.; Nicholson, J.K. Global metabolic profiling of animal and human tissues via UPLC-MS. Nat. Protoc. 2013, 8, 17-32. [CrossRef] [PubMed]

25. Huang, Q.; Tan, Y.X.; Yin, P.Y.; Ye, G.Z.; Gao, P.; Lu, X.; Wang, H.Y.; Xu, G.W. Metabolic characterization of hepatocellular carcinoma using nontargeted tissue metabolomics. Cancer Res. 2013, 73, 4994-5002. [CrossRef] [PubMed]

26. Human Metabolome Database (HMDB). Available online: http://www.hmdb.ca (accessed on 20 December 2017).

27. Metlin. Available online: http:/ / metlin.scripps.edu/ (accessed on 20 December 2017).

28. MassBank. Available online: http:/ / www.massbank.jp/ (accessed on 20 December 2017).

29. Lipidbank. Available online: http:/ / www.lipidbank.jp/ (accessed on 20 December 2017).

30. KEGG. Available online: http://www.genome.jp/kegg/ (accessed on 20 December 2017).

31. Liu, S.; Cai, W.; Wang, F.; Liu, Y.; Shang, Z.; Zhang, X.; Wang, Z.; Lu, J.; Zhang, J. UHPLC-LTQ-Orbitrap-based metabolomics coupled with metabolomics pathway analysis method for exploring the protection mechanism of Kudiezi injection in a rat anti-ischemic cerebral reperfusion damage model. Chin. J. Natl. Med. 2017, 15, 0955-0960. [CrossRef]

32. Liu, H.; Pu, H.; Yao, Q. A Study of Protective Effect of Taurine on Brain Cells with Ischemic Reperfusion Damage in Rats. Chin. J. Child Health 1999, 17, 196-197.

33. Yang, Z.; Li, P. Effect of Taurochenodeoxycholic Acid on Oxygen Free Radical Metabolism in Mice. Chin. Vet. Drug J. 2006, 40, 32-35.

34. Djuricic, B.; Olson, S.R.; Assaf, H.M.; Whittingham, T.S.; Lust, W.D.; Drewes, L.R. Formation of free choline in brain tissue during in vitro energy deprivation. J. Cerebr. Blood Flow Metab. 1991, 11, 308-313. [CrossRef] [PubMed]

35. Kozuka, M.; Iwata, N. Changes in levels of monoamines and their metabolites in incompletely ischemic brains of spontaneously hypertensive rats. Neurochem. Res. 1995, 20, 1429-1435. [CrossRef] [PubMed]

36. Longa, E.Z.; Weinstein, P.R.; Carlson, S.; Cummins, R. Reversible middle cerebral artery occlusion without craniectomy in rats. Stroke 1989, 20, 84-91. [CrossRef] [PubMed]

37. Tamura, A.; Graham, D.I.; McCulloch, J.; Teasdale, G.M. Focal cerebral ischaemia in the rat: 1. Description of technique and early neuropathological consequences following middle cerebral artery occlusion. J. Cereb. Blood Flow Metab. 1981, 1, 53-60. [CrossRef] [PubMed]

38. Dogan, A.; Tunc, E.; Ozturk, M.; Kerman, M.; Akhan, G. Electrocardiographic changes in patients with ischaemic stroke and their prognostic importance. Int. J. Clin. Pract. 2004, 58, 436-440. [CrossRef] [PubMed]

39. Joshia, C.N.; Jainb, S.K.; Murthy, P.S.R. An optimized triphenyltetrazolium chloride method for identification of cerebral infarcts. Brain Res. Protoc. 2004, 13, 11-17. [CrossRef] [PubMed]

40. Yan, X.L.; Li, H.; Bai, M.; Miao, M.S. Effect of total flavonoids of Radix Ilicis pubescentis on cerebral ischemia reperfusion model. Saudi J. Biol. Sci. 2017, 24, 595-602. [CrossRef] [PubMed]

41. Naz, S.; Vallejo, M.; García, A.; Barbas, C. Method validation strategies involved in non-targeted metabolomics. J. Chromatogr. A 2014, 1353, 99-105. [CrossRef] [PubMed] 
42. Chen, J.; Zhang, X.Y.; Cao, R.; Lu, X.; Zhao, S.M.; Fekete, A.; Huang, Q.; Schmitt Kopplin, P.; Wang, Y.S.; $\mathrm{Xu}$, Z.L. Serum 27-nor-5 beta-cholestane-3,7,12,24,25 pentol glucuronide discovered by metabolomics as potential diagnostic biomarker for epithelium ovarian cancer. J. Proteome Res. 2011, 10, 2625-2632. [CrossRef] [PubMed]

43. Liu, H.Y.; Zhang, L.; Zhao, B.S.; Zhang, Z.X.; Qin, L.L.; Zhang, Q.Q.; Wang, Q.; Lu, Z.W.; Gao, X.Y. Hypothalamus metabolomic profiling to elucidate the tissuetargeted biochemical basis of febrile response in yeast-induced pyrexia rats. Chem. Biol. Interact. 2015, 231, 61-70. [CrossRef] [PubMed]

44. Yin, P.Y.; Zhao, X.J.; Li, Q.R.; Wang, J.S.; Li, J.S.; Xu, G.W. Metabonomics study of intestinal fistulas based on ultraperformance liquid chromatography coupled with Q-TOF mass spectrometry (UPLC/Q-TOF MS). J. Proteome Res. 2006, 5, 2135-2143. [CrossRef] [PubMed]

Sample Availability: Samples of the compounds used in the study are available from the authors.

(C) 2018 by the authors. Licensee MDPI, Basel, Switzerland. This article is an open access article distributed under the terms and conditions of the Creative Commons Attribution (CC BY) license (http:/ / creativecommons.org/licenses/by/4.0/). 